\title{
Exploring life's sweet spot
}

\author{
Glycomics: like proteins and nucleic acids, carbohydrates have essential roles in the cell, but the \\ tools to synthesize and analyse this third class of biopolymer have, until recently, lagged far behind.
}

\section{Peter H. Seeberger}

There are three main classes of repeating biopolymers: nucleic acids, proteins and carbohydrates. DNA is the blueprint of life. It contains the information that is transferred from one generation to the next and acts as a template for the synthesis of RNA. RNA in turn mediates the production of proteins - abundant and versatile molecules that form structural components and catalyse most of the reactions in living cells.

One class of proteins, glycosyltransferases, carries out the synthesis of carbohydrates, whose roles vary from energy storage and supply to mechanical support. Small oligosaccharides are linked to proteins and lipids to form glycoproteins and glycolipids, which decorate the surface of most cells and play a role in cell adhesion and recognition. But unlike for nucleic acids and proteins, which have been the main focus of biomolecular research over the past 50 years, the tools required to advance the study of carbohydrates at a rapid pace have been largely missing.

The linearity of protein and nucleic-acid polymers and the regularity of the bonds joining each monomeric unit have allowed the development of efficient and reliable tools for their analysis and synthesis. But obtaining similar tools for carbohydrates has been hampered by their structural and chemical complexity. Each monosaccharide has several free hydroxyl groups that can be used to link the unit to the next monosaccharide. This allows branching and increases the number of possible polysaccharide structures. Synthetically, carbohydrates are challenging to work with because many more functional groups have to be protected to get one specific group to react, and the stereochemistry of every new glycosidic linkage needs to be controlled.

The new millennium has seen a concerted effort to develop the tools needed for glycomics. Improvements in massspectrometry techniques combined with the development of enzymatic and chemical tricks have made carbohydrate sequencing faster, more reliable and more widely applicable.

Better sequencing techniques allow glyco-structures in different cell populations to be compared and, with the help of synthesis, the relevant carbohydrates to be identified. After this improved molecular understanding of specific structures (or

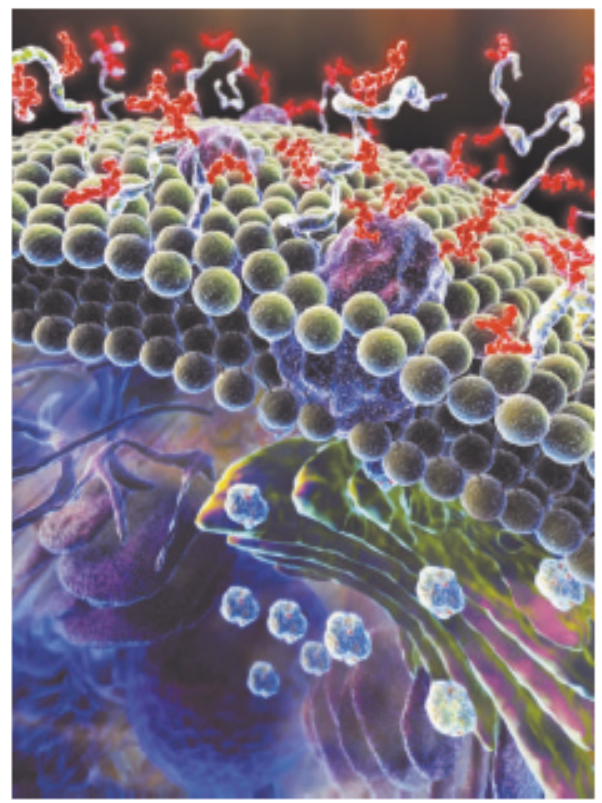

Sugars play a vital role in cell communication.

families of structures) is established, subsequent biological, biochemical and biophysical studies require defined molecules in much larger quantities. Our laboratory first reported the automated chemical synthesis of oligosaccharides in 2001. And although this process still cannot create all desired structures and is not yet available to the non-specialist, these goals are within reach.

Speedy access to sufficient quantities of defined carbohydrates allows the creation of tools that are commonplace in genomics and proteomics. Carbohydrate arrays that present synthetic content for high-throughput screening assays have been used to analyse interactions between carbohydrates and proteins, RNA and even whole cells. These sugar 'chips' have already yielded, for example, fingerprints of carbohydratebinding proteins.

Rapid screening of the interactions between carbohydrates and pathogens provides insights into the role of specific carbohydrates in the infectious process, which in turn may lead to new tools for disease diagnosis and prevention. Carbohydrate affinity columns are used to isolate proteins, and in vitro and in vivo imaging using labelled oligosaccharides has become possible using synthetic carbohydrate probes. New sequencing technologies coupled to the rapid synthesis of defined oligosaccharides and their analogues have also madeit possible to define carbohydrates involved in key signal-transduction events.
The glycomics advance that has perhaps had the broadest impact is that exploiting the induction of an immune response against unique pathogen or disease-specific oligosaccharides. This has lead to the creation of carbohydratebased vaccines against severe diseases including malaria, bacterial infections and cancer. Carbohydrate sequencing allows the oligosaccharide antigens to be identified and characterized, and chemical synthesis is then used to confirm antigen structure and prepare sufficient amounts of antigen to develop vaccines. Synthetic oligosaccharide vaccines against Haemophilis influenza type B have passed clinical trials; anticancervaccines are currently in dinical trials, and an anti-toxin malaria vaccine is in late preclinical development.

With improving automated synthesis and sequencing of carbohydrates, and a host of tools for glycomics in hand, the next few years should see researchers from all areas of the life sciences drawn to this rapidly developing field, and many more biotechnology opportunities created. As well as providing the basis of synthetic vaccines against bacterial and viral infections, and cancer, synthetic glycoproteins could overcome many of the problems and limitations currently encountered when using active glycoprotein therapeutics.

Defined heparin oligosaccharides are likely to become active agents not only in heart disease but also in cancer and other diseases. Glycolipid-based signalling processes will probably be exploited for therapeutic intervention in metabolic disease and for new immunomodulators. Eventually, synthetic biology should be able to supplement chemical efforts to create large quantities of defined carbohydrates for therapeutic and diagnostic applications. And no doubt new biological roles of carbohydrates will be discovered that surpass our wildest speculations.

Peter H. Seeberger is at the Laboratory for Organic Chemistry, Swiss Federal Institute of Technology (ETH), Wolfgang-PauliStrasse 10,8093 Zürich, Switzerland; and at the Burnham Institute, 10901 North Torrey Pines Road, La Jolla, California 92037, USA.

\section{FURTHER READING}

Varki, A. et al. (eds) Essentials of Glycobiology (Cold Spring Harbor Lab, New York, 1999).

Seeberger, P. H.Chem Comm.1715-1121(2003).

The Consortium for Functional Glycomics

mwwefunctionalghycomics.org 